\title{
Numerical Simulation of Temperature Field in Single-crystal Growth by Optical Floating Zone Technique
}

\author{
Meng-Jie SHI ${ }^{1, a}$, Yin-Zhou YAN ${ }^{1, b,{ }^{*}}$ \\ ${ }^{1}$ Institute of Laser Engineering, Beijing University of Technology, Beijing 100124, China \\ awzssmj@sina.com, byzyan@emails.bjut.edu.cn \\ ${ }^{*}$ Corresponding author
}

Keywords: FEM, Temperature Field, Optical Floating Zone, Single Crystal Growth.

\begin{abstract}
Optical floating zone (OFZ) technique is one of the most used methods for growth of single crystals. Although OFZ has been extensively investigated for past decades, the temperature distribution during growth has not been understood due to the complicated process during growth. It makes the optimization of growth parameters very difficult. In this work, we presented a simplified model to calculate the temperature field in floating zone by FEM. The optical properties of grown crystal and ceramic perform rod, lamp power, filament dimensions, ceramic rod diameter, floating zone dimensions, and ambient gas cooling rate were considered in the model. The calculated results show good agreement with the experiments. The developed model opens up a new way to optimize the growth parameters for a variety of single crystals.
\end{abstract}

\section{Introduction}

Man-made single crystal materials have widely been used in modern science and technology and the growth of crystals is an important way to obtain large size and high quality crystals. The growth techniques of single crystal materials include: solution growth method ${ }^{[1]}$, melt growth method ${ }^{[2,3]}$, gas phase growth method ${ }^{[4]}$, solid phase growth method ${ }^{[5]}$ etc. The optical floating zone method ${ }^{[6]}$

is one of the most studied methods due to the low costs, narrow molten zone and fast growth for oxide-based materials with high melting points ${ }^{[7-11]}$. However, the optimal parameters were only obtained by experiments due to the lack of understanding of growing process, which causes high-energy consumptions and time $\operatorname{costs}^{[12]}$. The rapid development of advanced numerical simulation based on modern computer technology provides a new tool for understanding of single crystal growing process and optimizing process parameters ${ }^{[13]}$.

Numerical simulation is based on the establishment of mathematical model in order to obtain the results difficult to capture from experiment. The process parameters and material properties can be tested in the model and the corresponding effects are hence observed. Then the simulated results can be applied to optimize the experimental procedures and process parameters in practice. In 1966, McCord developed the first commercial simulation software. It is the beginning of modern numerical simulation technology ${ }^{[14]}$. Arizumi and Kobayashi in the 70 s of last century opened on the crystal growth process of numerical simulation ${ }^{[15]}$. Nowadays, the commonly used in crystal numerical simulation methods include Monte Carlo method, boundary unit method, finite volume method, finite difference method, and finite element method. The most used ones are finite element method and the finite difference method ${ }^{[15-18]}$.

In this work, a FE model was developed to simulate the process of single crystal growth by optical floating zone technique. The optical properties of grown crystal and ceramic perform rod, lamp power, lamp filament dimensions, ceramic preform rod diameter, floating zone dimensions, and ambient gas cooling rate were considered in the model. The temperature fields during growth were simulated. The experiment with the same process parameters was also performed in order to validate 
the simulated results. According the numerical simulation, the growth parameters for $\mathrm{TiO}_{2}$ crystals were optimized, by which the high-quality $\mathrm{TiO}_{2}$ single crystal was grown.

\section{Theoretical Model and Experimental Procedure}

The growth system used in this work an optical floating zone furnace with four ellipsoidal mirrors (FZ-T-10000-H-VI-VP) with four halogen lamps of maximum power of $1.5 \mathrm{~kW}$ or $1 \mathrm{~kW}$, as Figure 1(a). The spectrum of the halogen lamp was measured by a Spectrometer (Spectra PR715), as shown in Figure 1(b). It can be seen that the main power intensity is located in the range of 500 2700nm and the central wavelength is around $1100 \mathrm{~nm}$.

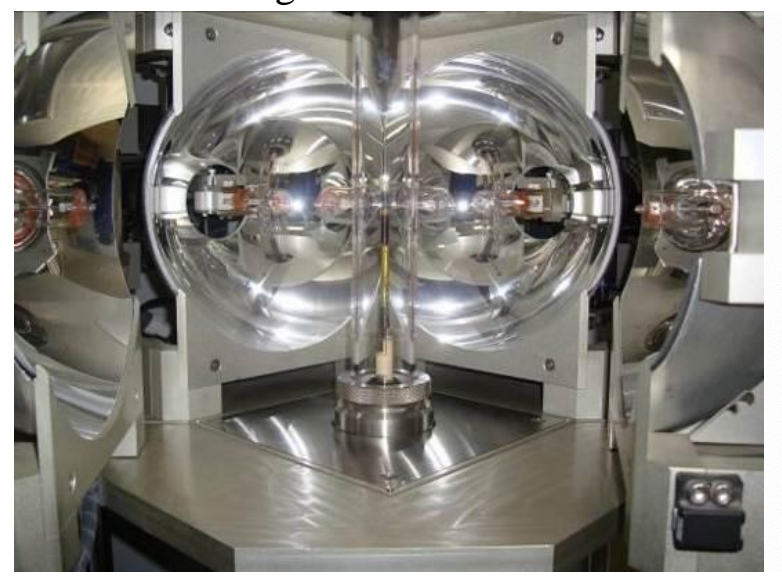

(a)

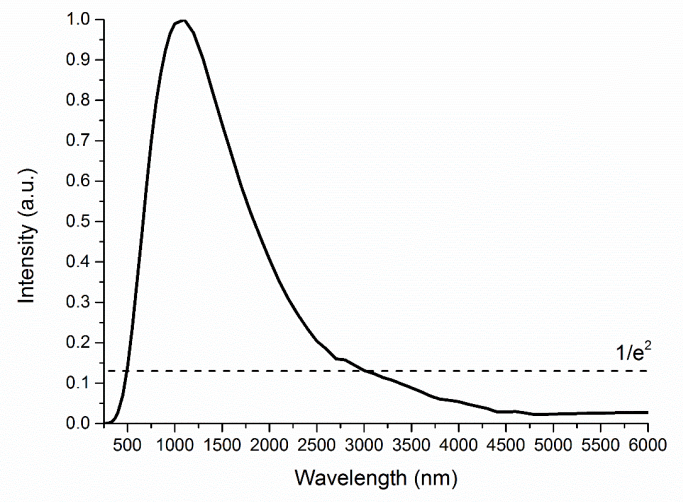

(b)

Fig.1 (a) The Optical Floating Zone Furnace and (b) Normalized Spectral Distribution of Halogen Lamp Used in This Work

In order to obtain the material absorption with respect to the lamp light within the main power distribution (500-2700nm), the reflectance and transmittance were measured by the spectrometer (UV-3600-230VCE). The top panel of Figure 2 demonstrates typical reflectance distribution of $\mathrm{TiO}_{2}$ perform rod, floating zone and crystal in the range of $500-2700 \mathrm{~nm}$. The transmittance is related to the thickness of the sample, the absorption coefficient $(\alpha)$ was therefore used to describe the absorption in different bands of the lamp light. The bottom panel of Figure 2 illustrates the absorption coefficients of the materials with respect to lamp wavelength.

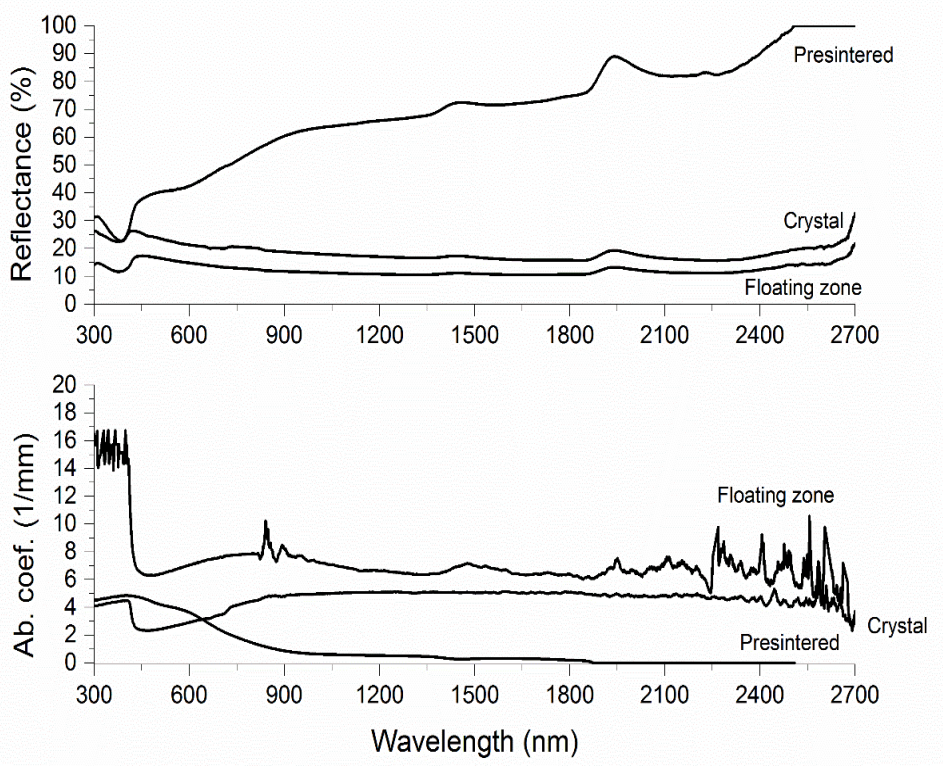

Fig.2 Reflectance and Absorption Coefficient of $\mathrm{TiO}_{2}$ Preform Rod, Floating Zone and Crystal 
Considering the light was focused by the ellipsoidal mirrors to the center of the furnace and perform rod rotation during growth, a 2D axisymmetric FE model was developed. The light energy caused heat generation rate distribution $(Q)$ can be described as

$$
Q=\frac{\beta \times(1-R) \times \eta^{4} \times P \times(l+w) \times \alpha}{2 \sqrt{\pi} l w h} e^{-\alpha(r(y)-x)-2 \frac{y^{2}}{\left(\frac{h}{2}\right)^{2}}}
$$

where $R$ is melting zone equivalent reflectance, $n$ is set percentage of lamp power, $a$ is floating zone equivalent penetration depth, $r$ is floating zone radius, $l, W$ and $h$ are halogen light filament length, width and height, $b$ is constant, $P$ is the maximum power of a single lamp.

The model was developed in ANSYS and calculated the corresponding temperature field. The experiments were also performed in order to validate the simulated results. The high purity (99.9\%) $\mathrm{TiO}_{2}$ powder was employed for growth of $\mathrm{TiO}_{2}$ single crystals. The powders and alcohol were first mixed together and milled for 24 hours. Then the milled powder was dried and put into a rubber for shaping by isostatic pressing at 30,000psi. The pressed green body was presentered at $1400^{\circ} \mathrm{C}$ with 10 hours in a vertical-type furnace. The sintered ceramic rod was cut and polished. In OFZ growth, the feed rate was $10-20 \mathrm{~mm} / \mathrm{h}$ and the rotation speeds of upper and lower rods were $30 \mathrm{rpm}$ and $20 \mathrm{rpm}$, respectively. The other parameters are exactly same as the numerical simulation used.

\section{Results and Discussion}

As an initial validation of the $\mathrm{FE}$ model, the effect of $\mathrm{TiO}_{2}$ ceramic rod diameter on power threshold for sample melting was calculated. Figure 3 shows the temperature fields with the lamp power thresholds for 4-mm- and 8-mm-diameter rod. It was found that the required power set percentages are $66.5 \%$ and $70 \%$ of $1500 \mathrm{~W}$, respectively, for the ceramic rods melting. The predicted values are in good agreement with the experimental results, i.e. $67 \%$ and $70 \%$.

The temperature gradient is another important factor affecting crystal quality in optical floating zone growth. The quality of grown crystal would be catastrophic when the axial and radial temperature gradients are improper. When the radial temperature gradient is low, the twin crystal is easily formed. However the high temperature gradient could lead to scattering particles and bubbles inside the crystal, causing the growing process breakdown. Therefore, the radial temperature gradient should be selected carefully. For lower axial temperature gradient, the crystallization and latent heat release are suppressed at solid-liquid interface, by which the crystal diameter cannot be uniform and the crystal quality is reduced. The high axial temperature gradient can solve above problems and stabilize the growing process.

According to numerical simulation, there are three parameters that can affect the temperature gradient, i.e. lamp power, lamp filament thickness, and perform rod diameter. Figure 4 shows the effect of lamp power on temperature gradient in floating zone. It can be seen that the axial and radial temperature gradients are both increased with the lamp power increasing. Therefore, the high lamp power is beneficial for high axial temperature gradient. Figure 5 demonstrates the temperature gradients using various lamp filament thicknesses. The axial and radial temperature gradients were reduced with a thicker filament. In order to obtain a steep axial temperature gradient, the thinner filament was recommended. Figure 6 shows the effect of ceramic rod diameter on temperature gradients. The axial temperature gradient was decreased with the diameter increasing whereas the radial one changed slightly. As a result, the small ceramic rod diameter should be applied for growth of high-quality crystal. 


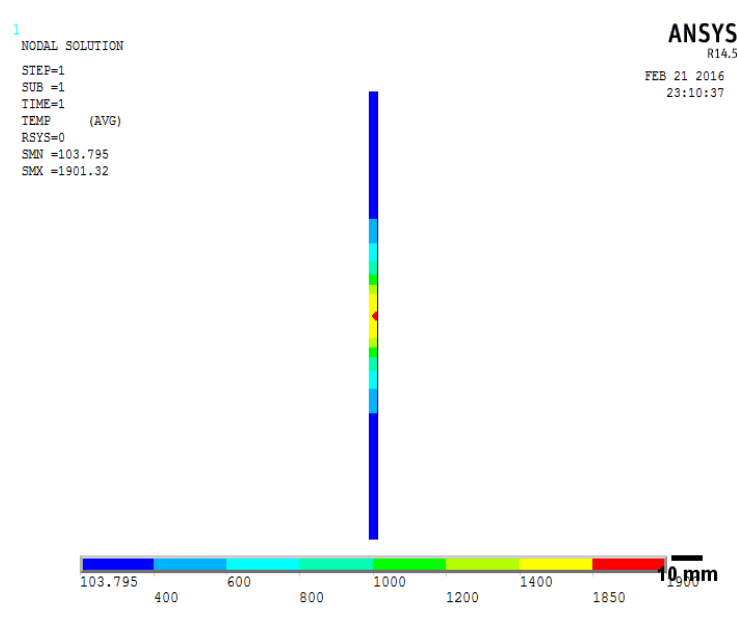

(a)

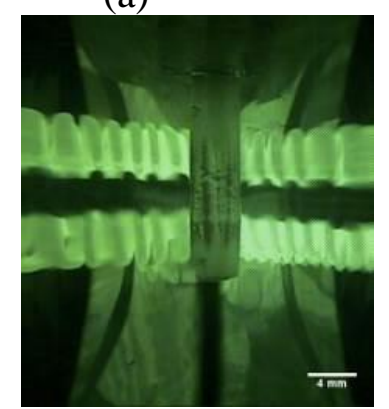

(c)

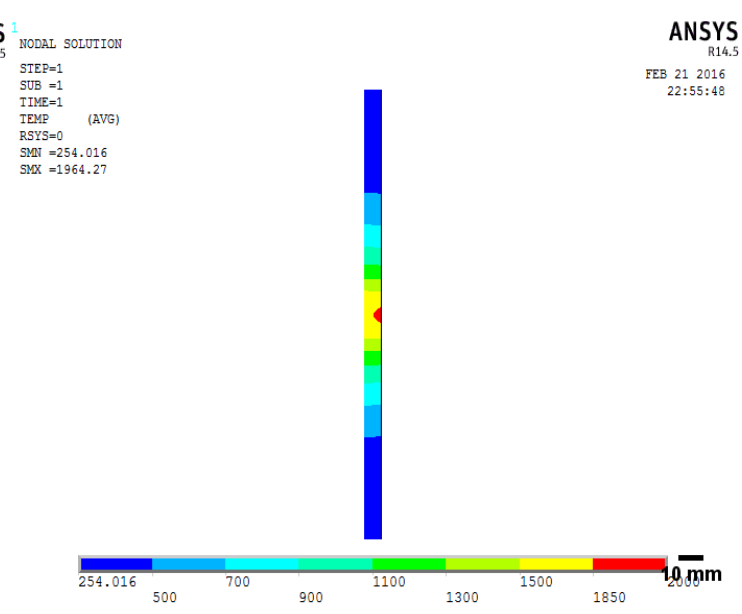

(b)

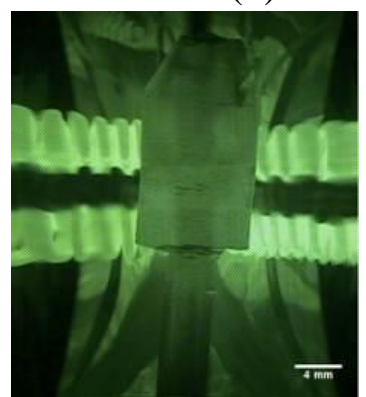

(d)

Fig.3 Temperature Fields Using the Threshold Lamp Powers for (a) 4-mm-diameter and (b) 8-mm-diameter $\mathrm{TiO}_{2}$ Ceramic Rods. (c)-(d) The Corresponding Experimental Results

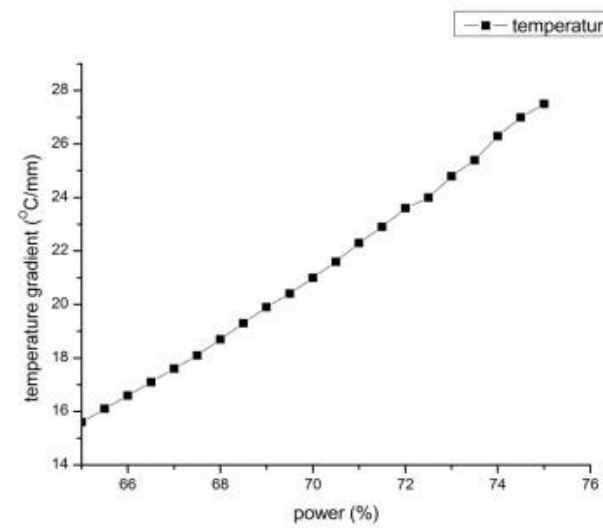

(a)

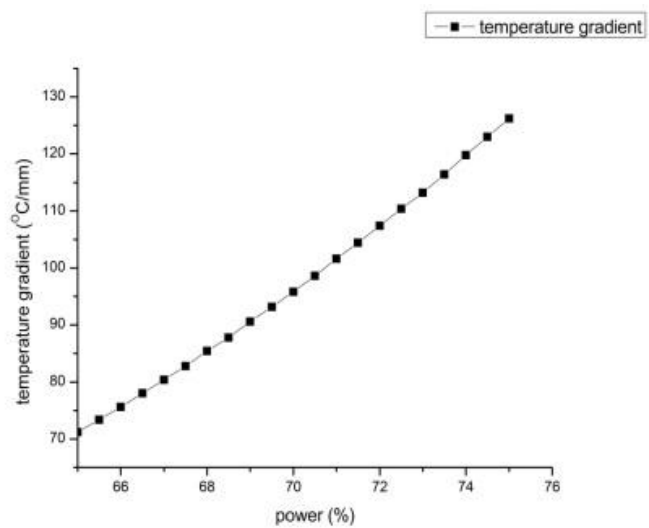

(b)

Fig.4 Effect of Lamp Power on Temperature Gradient in (a) Radial and (b) Axial Direction

In order to compare with the simulation results, the experiments with the same parameters were performed for $\mathrm{TiO}_{2}$ single crystal growth. Figure 7 shows the cross-sections of $\mathrm{TiO}_{2}$ crystals grown by different lamp powers. It can be seen that the scattering particles and bubbles and other impurities were reduced with the lamp power increasing, which indicated a high axial temperature gradient formed. The lamp filament thickness was also found to affect the floating zone, as shown in Figure 8. The thick filament caused a bowtie-shaped floating zone, where the melting was unstable and the liquid overflow could break the floating zone. However, the thin filament can stabilize the floating zone, by which the high quality crystal can be grown up. 


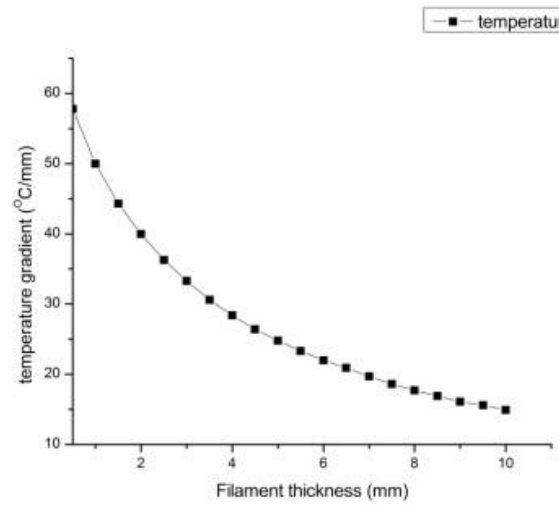

(a)

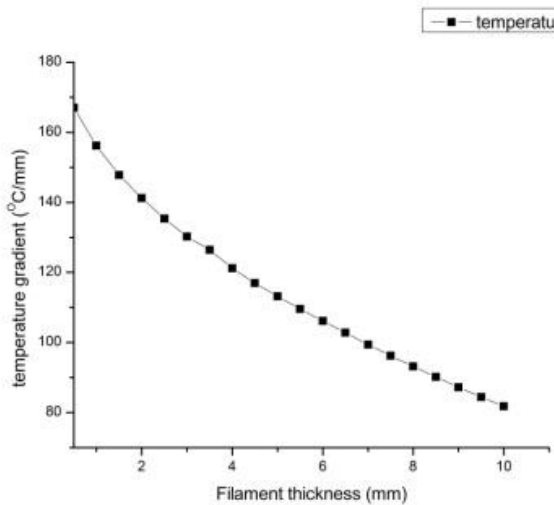

(b)

Fig.5 Effect of Lamp Filament Thickness on Temperature Gradient in (a) Radial and (b) Axial Direction

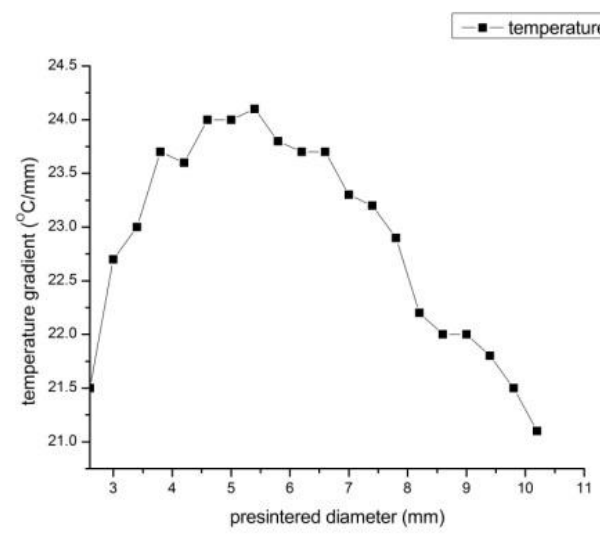

(a)

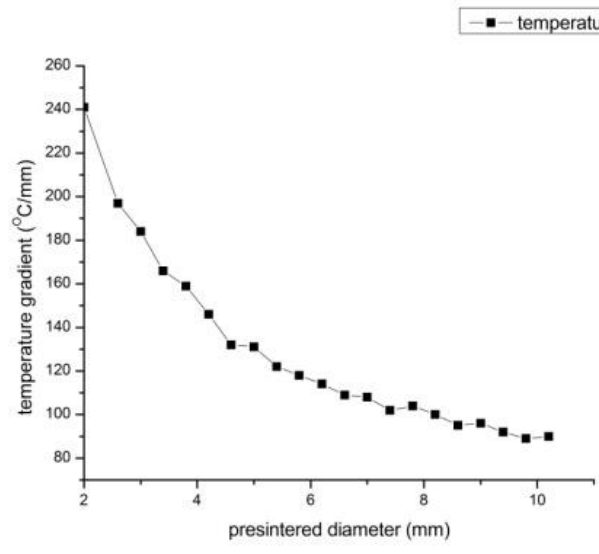

(b)

Fig.6 Effect of Ceramic Rod Diameter on Temperature Gradient in (a) Radial and (b) Axial Direction

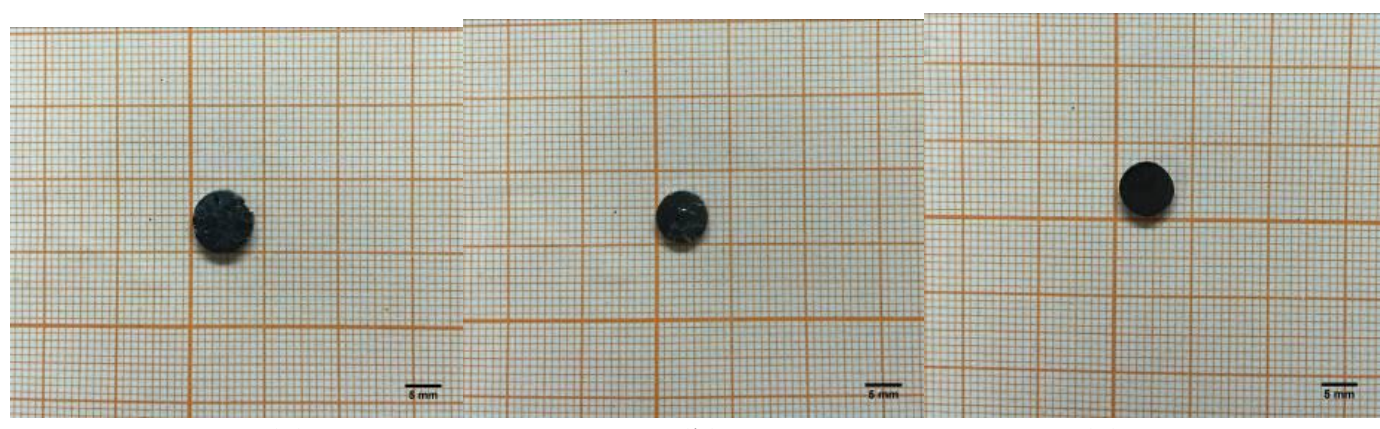

(a) (b) (c)

Fig.7 Cross-sections of $\mathrm{TiO}_{2}$ Crystals Grown by the Lamp Power of (a) $70.5 \%$, (b) $72 \%$ and (c) $73 \%$ of $1500 \mathrm{~W}$

The crystal quality was further examined by XRD swing curve, as shown in Figure 9. It can be found that only one peak existed in the $\mathrm{TiO}_{2}$ crystals grown by the optimized parameters and the corresponding FWHMs were very narrow. It indicates that the high quality of single crystal can be achieved by the above-mentioned optimized parameters. 


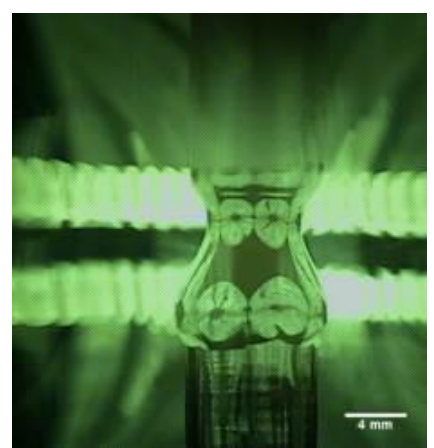

(a)

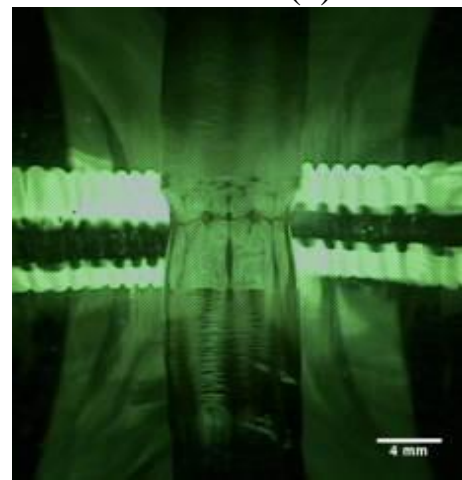

(c)

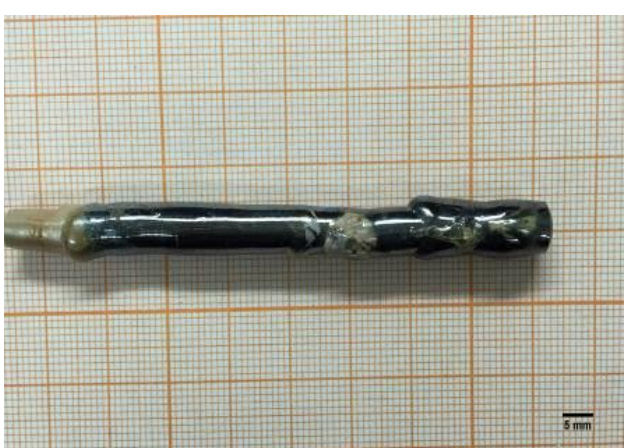

(b)

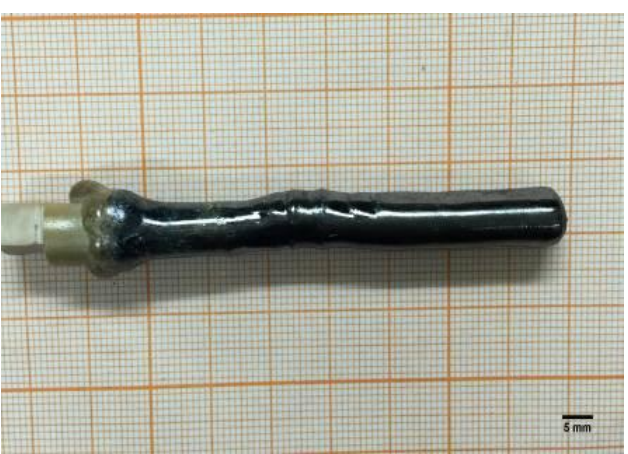

(d)

Fig. 8 The Effect of Lamp Filament Thickness on Floating Zone and Grown $\mathrm{TiO}_{2}$ Crystal (a) Floating Zone and (b) $\mathrm{TiO}_{2}$ Crystal Grown by 5.5-mm-thick Filament, and (c) Floating Zone and (d) $\mathrm{TiO}_{2}$ Crystal Grown by 4.6-mm-thick filament

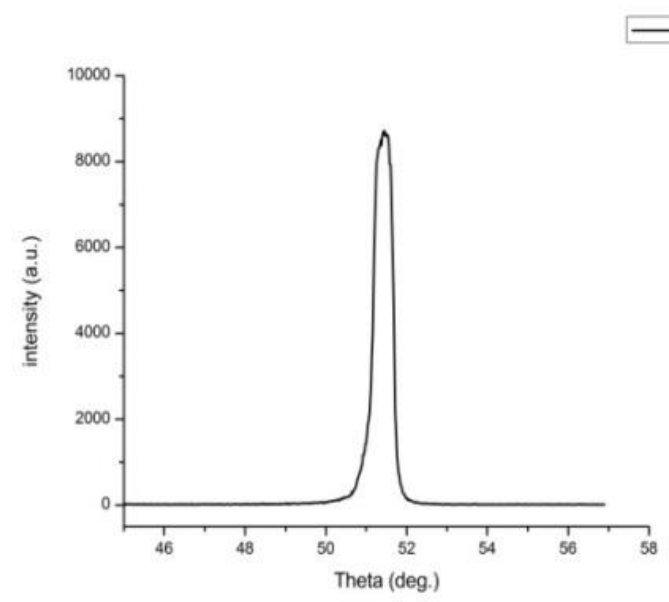

Fig.9 The XRD Swing Curves of TiO2 by Optimized Parameters

\section{Summary}

In this work, a FE model to simulate the temperature field in optical floating zone was developed. The optical properties of grown crystal and ceramic perform rod, lamp power, filament dimensions, ceramic preform rod diameter, floating zone dimensions, and ambient gas cooling rate were considered in the model. The numerical simulation indicated that the high lamp power (within a proper range), thin filament thickness and small perform rod were essential for high-quality single-crystal growth. The experiments were also performed to validate the prediction by numerical simulation. Such a work opens up a new way to optimize the parameters for growing a variety of high-quality single crystals by OFZ technique. 


\section{Acknowledgement}

The authors acknowledge the support of China Postdoctoral Science Foundation funded project (2014M560863).

\section{References}

[1] A.R Patel, A.V Rao. Growth of single crystals of $\mathrm{KClO}_{4}$ in silica gels [J]. Journal of Crystal Growth, 1977, 38(3):288-292.

[2] J Czochralski, A new method of measuring the speed of cristilation in metals. Zeitschrift FurPhysikalische Chemie. Stochiometrie Und Verwandtschaftslehre, 1917, Vo1.92 (2): 219221.

[3] P.W Bridgman, Certain Physical Properties of Single Crystals of Tungsten, Antimony, Bismuth, Tellurium, Cadmium, Zinc, and Tin. Proceedings of the American Academy of Arts and Sciences 60.6 (1925): 305-383.

[4] H. Maruska and J. Tietjen, THE PREPARATION AND PROPERTIES OFVAPOR-DEPOSITED SINGLE-CRYSTAL-LINE GaN. Appl. Phys. Lett.1 S (1969) 327.

[5] S Porowski. High pressure growth of GaN-new prospects for blue lasers [J]. Journal of Crystal Growth, 1996, 166(s 1-4):583-589.

[6] P.H Keck, M.J Golay. Crystallization of Silicon from a Floating Liquid Zone [J]. Physical Review, 1953, 89(6):1297-1297.

[7] S.U Wei, J.C Zhong, L.B Zhang, et al. Numerical Simulation Study on Field of Temperature During the Growth of Nd: YAG Crystal [J]. Chinese Journal of Lasers, 2003, 30(4):334-340.

[8] K Oka, H Unoki, H Shibata, et al. Crystal growth of rare-earth orthovanadate (RVO4) by the floating-zone method [J]. Journal of Crystal Growth, 2006, 286(2):288-293.

[9] M Higuchi, T Shimizu, J Takahashi, et al. Growth of RE: LuVO4 (RE=Nd, Tm, Yb) single crystals by the floating zone method and their spectroscopic properties [J]. Journal of Crystal Growth, 2005, 283(283):100-107.

[10] H Kimura, R Tanahashi, K Maiwa, et al. Crystal growth and characterization of ytterbium garnet and holmium garnet using the Fz technique [J]. Journal of Crystal Growth, 2009, 311(3):522-525.

[11] Gu, G.D Hucker, M Kim, Y.-J, et al. Single-crystal growth and superconductivity of (La1xSrx)2CaCu2O6+ $\delta[J]$. Journal of Crystal Growth, 2006, 287(2):318-322.

[12] Y.Q ZHENG, E SHI, L.W Wei, B.G WANG, et al. Research and Development of the Theories of Crystal Growth [J]. Journal of Inorganic Materials, 1999, 14(3):321-332.

[13] G.H Bruce, D.W Peaceman, Jr H H R, et al. Calculation of Unsteady-State Gas Flow through Porous Media [J]. Journal of Petroleum Technology, 1953, 5(3):79-92.

[14] N Kobayashi, T Arizumi. Computational analysis of the flow in a crucible [J]. Journal of Crystal Growth, 1975, 30(2):177-184.

[15] K.S Yee. Numerical solution of initial boundary value problems involving maxwell's equations in isotropic media [J]. Antennas \& Propagation IEEE Transactions on, 1966, 14(3):302-307.

[16] Y.R Li, D.F Ruan, N Imaishi, et al. Global simulation of a silicon Czochralski furnace in an axial magnetic field [J]. International Journal of Heat \& Mass Transfer, 2003, 46(15):2887-2898. 
[17] A Hayashi, M Kobayashi, C Jing, et al. Numerical simulation of the Czochralski growth process of oxide crystals with a relatively thin optical thickness [J]. International Journal of Heat \& Mass Transfer, 2004, 47(25):5501-5509.

[18] D Souptel, W Löser, G Behr. Vertical optical floating zone furnace: Principles of irradiation profile formation [J]. Journal of Crystal Growth, 2007, 300(2):538-550. 